\title{
Application of intuitionistic fuzzy sets to environmental management
}

\author{
I. M. Adamu \\ Department of Mathematics, Federal University Dutse \\ P.M.B 7156, Dutse, Jigawa State, Nigeria \\ e-mail: idreesmuhammadadam@gmail.com
}

Received: 24 April 2021

Accepted: 27 August 2021

\begin{abstract}
Environmental management is a decision making problem with a great deal of uncertainties. Intuitionistic fuzzy sets provide a model to elaborate these uncertainties and vagueness involve in decision making. This paper, proposes an application of intuitionistic fuzzy set to environmental management in order to determine the type of erosion(s) affecting some towns for an effective control measure that has to be taken using a distance function. In order to achieve this, a set of erosions and their causes are assumed, and also we assume a survey for the set of towns to determine the type of erosion approaching them for an effective control measure to be taken.
\end{abstract}

Keywords: Fuzzy sets, Intuitionistic fuzzy sets, Environmental management, Erosion.

2020 Mathematics Subject Classification: 20N20, 03E72.

\section{Introduction}

Most of the real-life situations are complex and to model them we need a simplification of the complex system. The simplification must be such that the information lost should be minimum. One way to do this is to allow some degree of uncertainty into it. To handle situations like this, Zadeh [39] proposed fuzzy sets. A fuzzy set has a membership function which assigns to each element of the universe of discourse, a number from the unit interval $[0,1]$ to indicate the degree of belongingness to the set under consideration. Fuzzy sets were introduced with a view to reconcile mathematical modelling and human knowledge in the engineering sciences. 
Atanassov [1,2] introduced a generalizatio of fuzzy sets called intuitionistic fuzzy sets. In the same time, a theory called intuitionistic fuzzy set theory was independently introduced by Takeuti and Titani [36] as a theory developed in (a kind of) intuitionistic logic, unrelated to Atanassov's. Atanassov's intuitionistic fuzzy sets provide a flexible framework to explain uncertainty and vagueness. Many researchers have confirmed the resourcefulness of IFSs in decision making problems due to its significance in tackling vagueness or the representation of imperfect knowledge. For the fundamentals and theory of IFSs, we recommend reading of $[3-13,19,28,29,40]$.

Most of the applications of IFSs are carried out using distance measures approach. Relevant literature for distance measures between intuitionistic fuzzy sets can be found in [8,31-33,37]. Distance measure between intuitionistic fuzzy sets is an important concept in fuzzy mathematics because of its wide applications in real world, such as in pattern recognition, machine learning, medical diagnostics, electoral systems, career determination, market prediction, among others, as presented in $[8,14,20,26,27,30,34,35,38]$. Ejegwa and Adamu [18] studied some distances between intuitionistic fuzzy sets of second type with application in medical diagnostics. Application of IFSs in career determination via an object-oriented approach was discussed in [22]. Several applications of IFSs using certain information measures have been discussed in $[15-17,23-25]$.

The notion of distances between IFS [31,33], which is very applicable in real-life situations, is the compelling motivation of this paper. In this paper, we study the idea of distances between IFS [31,33] and explore their application in Environmental Management to determine the erosion suffered by towns. We deploy the normalized Hamming distance for the application because it is the most reliable of the distance measures proposed in IFS, after a survey is conducted.

The paper is organized as follows: Section 2 provides some preliminaries on fuzzy sets, intuitionistic fuzzy sets and distance measures between intuitionistic fuzzy sets. We explore the application of intuitionistic fuzzy sets to Environmental Management in Section 3, while Section 4 concludes the paper and gives directions for future research work.

\section{Preliminaries}

We recall some basic notions of fuzzy sets and intuitionistic fuzzy sets.

\subsection{Fuzzy sets}

Definition 2.1 ([39]). Let $X$ be a nonempty set. A fuzzy set $A$ of $X$ is characterized by a membership function $\mu_{A}: X \rightarrow[0 ; 1]$, where:

$$
\mu_{A}(x)=\left\{\begin{aligned}
1, & \text { if } x \in X . \\
0, & \text { if } x \notin X . \\
(0,1) & \text { if } x \text { is partly in } X .
\end{aligned}\right.
$$

Alternatively, a fuzzy set $A$ of $X$ is an object having the form

$$
A=\left\{\left\langle x, \mu_{A}(x)\right\rangle \mid x \in X\right\} \text { or } A=\left\{\left\langle\frac{\mu_{A}(x)}{x}\right\rangle \mid x \in X\right\},
$$

where the function $\mu_{A}: X \rightarrow[0 ; 1]$ defines the degree of membership of the element $x \in X$. 
The closer the membership value $\mu_{A}$ to 1 , the more $x$ belongs to $A$, where the grades 1 and 0 represent full membership and full non-membership. In the classical set theory, the membership of elements to a set is assessed in binary terms according to a bivalent condition: an element either belongs or does not belong to the set. Classical bivalent sets are called in fuzzy set theory crisp sets.

Let us consider two examples of sets:

(i) All employees of $X Y Z$ who are over $1.8 \mathrm{~m}$ in height.

(ii) All employees of $X Y Z$ who are tall.

The first example is a classical set with a universe (all $X Y Z$ employees) and a membership rule that divides the universe into members (those over $1.8 \mathrm{~m}$ ) and non-members. The second example is a fuzzy set because some employees are definitely in the set and some are definitely not in the set, but some are borderline.

This distinction between the ins, the outs and the borderline is made more exact by the membership function $\mu$. If we return to our second example and let $A$ represent the fuzzy set of all tall employees and $x$ represent a member of the universe $X$ (i.e., all employees), then $\mu_{A}(x)$ would be $\mu_{A}(x)=1$ if $x$ is definitely tall or $\mu_{A}(x)=0$ if $x$ is definitely not tall or $0<\mu_{A}(x)<1$ for borderline cases.

\subsection{Intuitionistic fuzzy sets}

Definition 2.2 ([2]). Let a nonempty set $X$ be fixed. An IFS A of $X$ is an object having the form

$$
A=\left\{\left\langle x, \mu_{A}(x), \nu(x)\right\rangle \mid x \in X\right\}
$$

or

$$
A=\left\{\left\langle\frac{\mu_{A}(x), \nu(x)}{x}\right\rangle \mid x \in X\right\},
$$

where the functions $\mu_{A}: X \rightarrow[0 ; 1]$ and $\nu_{A}: X \rightarrow[0 ; 1]$ define the degree of membership and the degree of non-membership, respectively, of the element $x \in X$ to A which is a subset of $X$, and for every $x \in X$

$$
0 \leq \mu_{A}(x)+\nu_{A}(x) \leq 1
$$

For each $A$ in $X$,

$$
\pi_{A}(x)=1-\mu_{A}(x)-\nu_{A}(x)
$$

is the intuitionistic fuzzy set index or hesitation margin of $x$ in $X$. The hesitation margin $\pi_{A}(x)$ is the degree of indeterminacy of the belonging of $x \in X$ to the set $A$ and $\pi_{A}(x) \in[0,1]$. The hesitation margin is the function that expresses lack of knowledge of whether $x \in X$ or $x \notin X$.

Thus,

$$
\mu_{A}(x)+\nu_{A}(x)+\pi_{A}(x)=1 .
$$

Example 2.3. Let $X=\{x, y, z\}$ be a fixed universe of discourse and

$$
A=\left\{\left\langle\frac{0.7,0.2}{x}\right\rangle,\left\langle\frac{0.6,0.2}{y}\right\rangle,\left\langle\frac{0.4,0.3}{z}\right\rangle\right\}
$$

be an intuitionistic fuzzy set of $X$. The hesitation margins of the elements $x, y, z$ to $A$ are

$$
\pi_{A}(x)=0.1, \pi_{A}(y)=0.2 \text { and } \pi_{A}(z)=0.3 .
$$




\subsection{Distances between intuitionistic fuzzy sets}

Definition 2.3 ([31,33]). Let $A, B, C \in I F S(X)$. Then, the distance measure d between the IFSs is a function $d: I F S \times I F S \rightarrow[0,1]$ satisfying:

(i) $0 \leq d(A, B) \leq 1$ (boundedness)

(ii) $d(A, B)=0$ iff $A=B$ (separability)

(iii) $d(A, B)=d(B, A)$ (symmetry)

(iv) $d(A, C)+d(B, C) \geq d(A, B)$ (triangle inequality).

For any two IFS $A$ and $B$ of $X=\left\{X_{1}, \ldots, X_{n}\right\}$ the following distance measures have been proposed:

(i) Hamming distance (HD)

$$
d_{I F S}(A, B)_{H D}=\frac{1}{2} \sum_{i=1}^{n}\left[\left|\mu_{A}\left(x_{i}\right)-\mu_{B}\left(x_{i}\right)\right|+\left|\nu_{A}\left(x_{i}\right)-\nu_{B}\left(x_{i}\right)\right|+\left|\pi_{A}\left(x_{i}\right)-\pi_{B}\left(x_{i}\right)\right|\right]
$$

(ii) Euclidean distance (ED)

$$
d_{I F S}(A, B)_{E D}=\left(\frac{1}{2} \sum_{i=1}^{n}\left[\left(\mu_{A}\left(x_{i}\right)-\mu_{B}\left(x_{i}\right)\right)^{2}+\left(\nu_{A}\left(x_{i}\right)-\nu_{B}\left(x_{i}\right)\right)^{2}+\left(\pi_{A}\left(x_{i}\right)-\pi_{B}\left(x_{i}\right)\right)^{2}\right]\right)^{\frac{1}{2}}
$$

(iii) normalized Hamming distance (nHD)

$$
d_{I F S}(A, B)_{n H D}=\frac{1}{2 n} \sum_{i=1}^{n}\left[\left|\mu_{A}\left(x_{i}\right)-\mu_{B}\left(x_{i}\right)\right|+\left|\nu_{A}\left(x_{i}\right)-\nu_{B}\left(x_{i}\right)\right|+\left|\pi_{A}\left(x_{i}\right)-\pi_{B}\left(x_{i}\right)\right|\right]
$$

(iv) normalized Euclidean distance (nED)

$d_{I F S}(A, B)_{n E D}=\left(\frac{1}{2 n} \sum_{i=1}^{n}\left[\left(\mu_{A}\left(x_{i}\right)-\mu_{B}\left(x_{i}\right)\right)^{2}+\left(\nu_{A}\left(x_{i}\right)-\nu_{B}\left(x_{i}\right)\right)^{2}+\left(\pi_{A}\left(x_{i}\right)-\pi_{B}\left(x_{i}\right)\right)^{2}\right]\right)^{\frac{1}{2}}$

for

$$
\pi_{A}\left(x_{i}\right)=1-\mu_{A}\left(x_{i}\right)-\nu_{A}\left(x_{i}\right)
$$

and

$$
\pi_{B}\left(x_{i}\right)=1-\mu_{B}\left(x_{i}\right)-\nu_{B}\left(x_{i}\right) .
$$

All these distances have been tested for reliability with the normalized Hamming distance as the most reliable one as it is shown in the following example.

Example 3.5. Let us consider the following intuitionistic fuzzy sets $A, B$ and $C$ of $X=$ $\{1,2,3,4,5,6,7\}$.

$$
\begin{aligned}
& A=\left\{\left\langle\frac{0.7,0.2,0.3}{1}\right\rangle,\left\langle\frac{0.6,0.2,0.2}{2}\right\rangle,\left\langle\frac{0.4,0.3,0.3}{4}\right\rangle,\left\langle\frac{0.4,0.1,0.5}{5}\right\rangle,\left\langle\frac{0.8,0.2,0.0}{6}\right\rangle\right\}, \\
& B=\left\{\left\langle\frac{1.0,0.0,0.0}{1}\right\rangle,\left\langle\frac{0.3,0.3,0.4}{4}\right\rangle,\left\langle\frac{0.5,0.2,0.3}{5}\right\rangle,\left\langle\frac{0.4,0.0,0.6}{7}\right\rangle\right\}, \\
& C=\left\{\left\langle\frac{0.2,0.7,0.3}{1}\right\rangle,\left\langle\frac{0.6,0.1,0.3}{2}\right\rangle,\left\langle\frac{0.4,0.3,0.3}{3}\right\rangle,\left\langle\frac{0.5,0.1,0.4}{4}\right\rangle,\left\langle\frac{0.7,0.2,0.1}{6}\right\rangle\right\} .
\end{aligned}
$$


Applying the distances between IFS, we obtain the following results:

$$
\begin{aligned}
d_{I F S}(A, B)_{H D}= & \frac{1}{2} \sum_{i=1}^{7}[|0.7-1.0|+|0.2-0.0|+|0.3-0.0|+|0.6-0.0|+|0.2-0.0|+|0.2-0.0| \\
& +|0.4-0.3|+|0.3-0.3|+|0.3-0.4|+|0.4-0.5|+|0.1-0.2|+|0.5-0.3| \\
& \quad+|0.8-0.0|+|0.2-0.0|+|0.0-0.0|+|0.0-0.4|+|0.0-0.0|+|0.0-0.6|] \\
= & 2.20
\end{aligned}
$$

$$
\begin{aligned}
d_{I F S}(A, C)_{H D}= & \frac{1}{2} \sum_{i=1}^{7}[|0.7-0.2|+|0.2-0.7|+|0.3-0.3|+|0.6-0.6|+|0.2-0.1|+|0.2-0.3| \\
& \quad+|0.0-0.4|+|0.0-0.3|+|0.0-0.3|+|0.4-0.5|+|0.3-0.1|+|0.3-0.4| \\
& \quad+|0.4-0.0|+|0.1-0.0|+|0.5-0.0|+|0.8-0.7|+|0.2-0.2|+|0.0-0.1|] \\
= & 1.90
\end{aligned}
$$$$
d_{I F S}(B, C)_{H D}=\frac{1}{2} \sum_{i=1}^{7}[|1.0-0.2|+|0.0-0.7|+|0.0-0.3|+|0.0-0.6|+|0.0-0.1|+|0.0-0.3|
$$$$
+|0.0-0.4|+|0.0-0.3|+|0.0-0.3|+|0.3-0.5|+|0.3-0.1|+|0.4-0.4|
$$$$
+|0.5-0.0|+|0.2-0.0|+|0.3-0.0|+|0.0-0.7|+|0.2-0.2|+|0.0-0.1|
$$$$
+|0.4-0.0|+|0.0-0.0|+|0.4-0.0|]
$$$$
=3.30 \text {. }
$$

$$
\begin{aligned}
d_{I F S}(A, B)_{E D}=( & \frac{1}{2} \sum_{i=1}^{7}\left[(0.7-1.0)^{2}+(0.2-0.0)^{2}+(0.3-0.0)^{2}+(0.6-0.0)^{2}+(0.2-0.0)^{2}\right. \\
& +(0.2-0.0)^{2}+(0.4-0.3)^{2}+(0.3-0.3)^{2}+(0.3-0.4)^{2}+(0.4-0.5)^{2} \\
& +(0.1-0.2)^{2}+(0.5-0.3)^{2}+(0.8-0.0)^{2}+(0.2-0.0)^{2}+(0.0-0.0)^{2} \\
& \left.\left.+(0.0-0.4)^{2}+(0.0-0.0)^{2}+(0.0-0.6)^{2}\right]\right)^{\frac{1}{2}} \\
= & 0.97
\end{aligned}
$$

$$
\begin{aligned}
d_{I F S}(A, C)_{E D}=( & \frac{1}{2} \sum_{i=1}^{7}\left[(0.7-0.2)^{2}+(0.2-0.7)^{2}+(0.3-0.3)^{2}+(0.6-0.6)^{2}+(0.2-0.1)^{2}\right. \\
& +(0.2-0.3)^{2}+(0.0-0.4)^{2}+(0.0-0.3)^{2}+(0.0-0.3)^{2}+(0.4-0.5)^{2} \\
& +(0.3-0.1)^{2}+(0.3-0.4)^{2}+(0.4-0.0)^{2}+(0.1-0.0)^{2}+(0.5-0.0)^{2} \\
& \left.\left.+(0.8-0.7)^{2}+(0.2-0.2)^{2}+(0.0-0.1)^{2}\right]\right)^{\frac{1}{2}} \\
= & 1.14
\end{aligned}
$$$$
\begin{aligned}
d_{I F S}(B, C)_{E D}=( & \frac{1}{2} \sum_{i=1}^{7}\left[(0.1-0.2)^{2}+(0.0-0.7)^{2}+(0.0-0.3)^{2}+(0.0-0.6)^{2}+(0.0-0.1)^{2}\right. \\
& +(0.0-0.3)^{2}+(0.0-0.4)^{2}+(0.0-0.3)^{2}+(0.0-0.3)^{2}+(0.3-0.5)^{2} \\
& +(0.3-0.1)^{2}+(0.4-0.4)^{2}+(0.5-0.0)^{2}+(0.2-0.0)^{2}+(0.3-0.0)^{2} \\
& +(0.0-0.7)^{2}+(0.0-0.2)^{2}+(0.0-0.1)^{2}+(0.7-0.0)^{2}+(0.0-0.0)^{2} \\
& \left.\left.+(0.6-0.0)^{2}\right]\right)^{\frac{1}{2}}
\end{aligned}
$$$$
=1.27 \text {. }
$$ 


$$
\begin{aligned}
d_{I F S}(A, B)_{n H D}= & \frac{1}{14} \sum_{i=1}^{7}[|0.7-1.0|+|0.2-0.0|+|0.3-0.0|+|0.6-0.0|+|0.2-0.0|+|0.2-0.0| \\
& +|0.4-0.3|+|0.3-0.3|+|0.3-0.4|+|0.4-0.5|+|0.1-0.2|+|0.5-0.3| \\
& +|0.8-0.0|+|0.2-0.0|+|0.0-0.0|+|0.0-0.4|+|0.0-0.0|+|0.0-0.6|] \\
= & 0.34
\end{aligned}
$$

$$
\begin{aligned}
d_{I F S}(A, C)_{n H D}= & \frac{1}{14} \sum_{i=1}^{7}[|0.7-0.2|+|0.2-0.7|+|0.3-0.3|+|0.6-0.6|+|0.2-0.1|+|0.2-0.3| \\
& +|0.0-0.4|+|0.0-0.3|+|0.0-0.3|+|0.4-0.5|+|0.3-0.1|+|0.3-0.4| \\
& +|0.4-0.0|+|0.1-0.0|+|0.5-0.0|+|0.8-0.7|+|0.2-0.2|+|0.0-0.1|] \\
= & 0.27
\end{aligned}
$$

$$
\begin{aligned}
d_{I F S}(B, C)_{n H D}=\frac{1}{14} & \sum_{i=1}^{7}[|0.1-0.2|+|0.0-0.7|+|0.0-0.3|+|0.0-0.6|+|0.0-0.1|+|0.0-0.3| \\
& +|0.0-0.4|+|0.0-0.3|+|0.0-0.3|+|0.3-0.5|+|0.3-0.1|+|0.4-0.4| \\
& +|0.5-0.0|+|0.2-0.0|+|0.3-0.0|+|0.0-0.7|+|0.0-0.2|+|0.0-0.1| \\
& +|0.4-0.0|+|0.0-0.0|+|0.6-0.0|] \\
= & 0.41
\end{aligned}
$$

$$
\begin{aligned}
d_{I F S}(A, B)_{n E D}=( & \frac{1}{14} \sum_{i=1}^{7}\left[(0.7-1.0)^{2}+(0.2-0.0)^{2}+(0.3-0.0)^{2}+(0.6-0.0)^{2}+(0.2-0.0)^{2}\right. \\
& +(0.2-0.0)^{2}+(0.4-0.3)^{2}+(0.3-0.3)^{2}+(0.3-0.4)^{2}+(0.4-0.5)^{2} \\
& +(0.1-0.2)^{2}+(0.5-0.3)^{2}+(0.8-0.0)^{2}+(0.2-0.0)^{2}+(0.0-0.0)^{2} \\
& \left.\left.+(0.0-0.4)^{2}+(0.0-0.0)^{2}+(0.0-0.6)^{2}\right]\right)^{\frac{1}{2}} \\
= & 0.42
\end{aligned}
$$

$$
\begin{aligned}
d_{I F S}(A, C)_{n E D}= & \frac{1}{14} \sum_{i=1}^{7}\left[(0.7-0.2)^{2}+(0.2-0.7)^{2}+(0.3-0.3)^{2}+(0.6-0.6)^{2}+(0.2-0.1)^{2}\right. \\
+ & (0.2-0.3)^{2}+(0.0-0.4)^{2}+(0.0-0.3)^{2}+(0.0-0.3)^{2}+(0.4-0.5)^{2} \\
& \quad+(0.3-0.1)^{2}+(0.3-0.4)^{2}+(0.4-0.0)^{2}+(0.1-0.0)^{2}+(0.5-0.0)^{2} \\
& \left.\left.\quad+(0.8-0.7)^{2}+(0.2-0.2)^{2}+(0.0-0.1)^{2}\right]\right)^{\frac{1}{2}} \\
= & 0.43 .
\end{aligned}
$$$$
\begin{aligned}
d_{I F S}(B, C)_{n E D}= & \frac{1}{14} \sum_{i=1}^{7}\left[(0.1-0.2)^{2}+(0.0-0.7)^{2}+(0.0-0.3)^{2}+(0.0-0.6)^{2}+(0.0-0.1)^{2}\right. \\
+ & (0.0-0.3)^{2}+(0.0-0.4)^{2}+(0.0-0.3)^{2}+(0.0-0.3)^{2}+(0.3-0.5)^{2} \\
& +(0.3-0.1)^{2}+(0.4-0.4)^{2}+(0.5-0.0)^{2}+(0.2-0.0)^{2}+(0.3-0.0)^{2} \\
& +(0.0-0.7)^{2}+(0.0-0.2)^{2}+(0.0-0.1)^{2}+(0.4-0.0)^{2}+(0.0-0.0)^{2} \\
& \left.\left.+(0.6-0.0)^{2}\right]\right)^{\frac{1}{2}} \\
= & 0.49
\end{aligned}
$$ 
Table 1 gives the summary of the results.

\begin{tabular}{|c|cccc|}
\hline Distances & $H D$ & $E D$ & $n H D$ & $n E D$ \\
\hline$d(A, B)$ & 2.20 & 0.98 & 0.34 & 0.43 \\
$d(A, C)$ & 1.90 & 1.14 & 0.27 & 0.43 \\
$d(B, C)$ & 3.30 & 1.27 & 0.41 & 0.49 \\
\hline
\end{tabular}

Table 1. Calculated distances under different metrics

From Table 1, we observe that:

(i) $d(A, B) \in[0,1]$ for Euclidean, $n$-Hamming and $n$-Euclidean distances distances.

(ii) $d(A, C), d(B, C) \in[0,1]$ for $n$-Hamming and $n$-Euclidean distances.

Notwithstanding, the $n$-Hamming distance is the most reasonable/efficient of the distance measures discussed. Hence, we adopt the $n$-Hamming distance for application to environmental management.

\section{Application of intuitionistic fuzzy sets to environmental management}

In this section, we present an application of IFS in environmental management. In a given survey, suppose $C$ is a set of causes, $E$ is a set of erosion types, and $T$ is a set of towns. Using environmental knowledge of erosion and its causes, we know that some causes are more peculiar to a particular erosion than to other, that is, each erosion has a peculiar cause which reveals a degree of association, $\mu$, and a degree of non-association, $\nu$, between the cause and erosion. Now, we discuss the concept of IFS in environmental management. The methodology involves mainly three stages viz:

(i) determination of cause;

(ii) formulation of environmental knowledge based on IFS values;

(iii) determination of management using $n$-Hamming distance between $T$ and $E$.

Then, the distance $d$ between towns and erosion with respect to causes is given as

$$
d_{I F S}(T, E)_{n H D}=\frac{1}{2 n} \sum_{i=1}^{n}\left[\left|\mu_{T}\left(c_{i}\right)-\mu_{E}\left(c_{i}\right)\right|+\left|\nu_{T}\left(c_{i}\right)-\nu_{E}\left(c_{i}\right)\right|+\left|\pi_{T}\left(c_{i}\right)-\pi_{E}\left(c_{i}\right)\right|\right]
$$

where $c_{i} \in C$, and $n$ is the number of causes. A town is said to suffer from a particular erosion if the distance, $d$ between the causes and the town is the least. To see the application of the method, let us make a hypothetical case.

\subsection{Experimental example}

Suppose there are five towns, $T$ given as a set $T=\left\{T_{1}, T_{2}, T_{3}, T_{4}\right\}$ to be surveyed for the set of erosion types given as:

$$
E=\{\text { water erosion, gully erosion, tunnel erosion, wind erosion }\},
$$


which have the set of causes:

$$
C=\{\text { cultivation, grazing, improper design, runoff water, poor vegetation }\}
$$

From the survey knowledge, Table 2 contains each erosion and its causes in IFS values.

\begin{tabular}{|c|ccccc|}
\hline & Cultivation & Grazing & Improper design & Runoff water & Poor vegetation \\
\hline water erosion & $(0.8,0.1,0.1)$ & $(0.7,0.2,0.1)$ & $(0.3,0.5,0.2)$ & $(0.5,0.3,0.2)$ & $(0.5,0.4,0.1)$ \\
wind erosion & $(0.2,0.7,0.1)$ & $(0.9,0.0,0.1)$ & $(0.7,0.2,0.1)$ & $(0.6,0.3,0.1)$ & $(0.7,0.2,0.1)$ \\
gully erosion & $(0.5,0.3,0.2)$ & $(0.3,0.5,0.2)$ & $(0.2,0.7,0.1)$ & $(0.2,0.6,0.2)$ & $(0.4,0.4,0.2)$ \\
tunnel erosion & $(0.1,0.7,0.2)$ & $(0.3,0.6,0.1)$ & $(0.8,0.1,0.1)$ & $(0.1,0.8,01)$ & $(0.1,0.8,0.1)$ \\
\hline
\end{tabular}

Table 2. Erosion vs Causes

In Table 2 , each cause $C_{i}$ is described by three numbers, i.e., membership $\mu$, non-membership $\nu$ and hesitation margin $\pi$.

For the sake of control measure, we assumed that the surveyed results were collected from the towns and analyzed, the obtained results are presented in Table 2.

\begin{tabular}{|c|ccccc|}
\hline & Cultivation & Grazing & Improper design & Runoff water & Poor vegetation \\
\hline$T_{1}$ & $(0.6,0.2,0.2)$ & $(0.4,0.4,0.2)$ & $(0.2,0.7,0.1)$ & $(0.5,0.3,0.3)$ & $(0.3,0.5,0.2)$ \\
$T_{2}$ & $(0.3,0.5,0.2)$ & $(0.6,0.2,0.2)$ & $(0.5,0.3,0.2)$ & $(0.4,0.5,0.1)$ & $(0.7,0.1,0.2)$ \\
$T_{3}$ & $(0.2,0.6,0.2)$ & $(0.3,0.3,0.4)$ & $(0.7,0.1,0.2)$ & $(0.3,0.6,0.1)$ & $(0.3,0.5,0.2)$ \\
$T_{4}$ & $(0.4,0.4,0.2)$ & $(0.4,0.2,0.4)$ & $(0.1,0.6,0.3)$ & $(0.5,0.4,0.1)$ & $(0.4,0.5,0.1)$ \\
\hline
\end{tabular}

Table 3. Towns vs Causes

Using Eq. (1), which is the normalized Hamming distance aforementioned, to calculate the distance between each of the towns in Table 3 and each of the erosions in Table 1 with respect to each of the causes, we get the following tables as shown below.

\begin{tabular}{|l|cccc|}
\hline & Water erosion & Wind erosion & Gully erosion & Tunnel erosion \\
\hline$T_{1}$ & 0.29 & 0.53 & 0.18 & 0.54 \\
$T_{2}$ & 0.26 & 0.25 & 0.30 & 0.48 \\
$T_{3}$ & 0.48 & 0.38 & 0.33 & 0.25 \\
$T_{4}$ & 0.26 & 0.45 & 0.25 & 0.53 \\
\hline
\end{tabular}

Table 4. Distance between Towns vs Erosions

From Table 4, town $T_{1}$ is to be managed from Gully erosion, town $T_{2}$ is to be managed from Wind erosion, town $T_{3}$ is to be managed from Tunnel erosion and town $T_{4}$ is to be managed from Gully erosion. 


\section{Conclusion}

The paper presents the application of intuitionistic fuzzy sets to environmental management in order to determine the type of erosion affecting some towns for an effective management to be taken, using a distance function. In order to achieve this, a set of erosions and their causes are assumed, and also we assume a survey for the set of towns is conducted to determine the type of erosion approaching them for an effective control measure to be taken. The distances between intuitionistic fuzzy sets presented in this work could be applied to many other real-life problems involving decision making under uncertainty.

\section{References}

[1] Atanassov, K. (1983). Intuitionistic Fuzzy sets. VII ITKR Session, Bulgarian: Central Science-Technical Academy of Science, 1697/84.

[2] Atanassov, K. T. (1986). Intuitionistic fuzzy sets. Fuzzy sets and Systems, 20(1), 87-96.

[3] Atanassov, K. T. (1988). Review and new results on intuitionistic fuzzy sets. Preprint IM-MFAIS-1-88, Sofia.

[4] Atanassov, K. T. (1989). More on intuitionistic fuzzy sets. Fuzzy Sets and Systems, 33(1), 37-46.

[5] Atanassov, K. T. (1991). Temporal intuitionistic fuzzy sets. Compt. Rend. Acad. Bulgare. Sci., 44(7), 5-7.

[6] Atanassov, K. T. (1992). Remark on the intuitionistic fuzzy sets. Fuzzy Sets and Systems, 51(1), 117-118.

[7] Atanassov, K. T. (1994). New operations defined over Intuitionistic fuzzy sets. Fuzzy Sets and Systems, 61(2), 137-142.

[8] Atanassov, K. T. (1999). Intuitionistic Fuzzy Sets: Theory and Applications, Physica-Verlag, Heidelberg.

[9] Atanassov, K. T. (2003). Intuitionistic fuzzy sets - Past, present, and future. 3rd Conference of the European Society for Fuzzy Logic and Technology, Zittau, Germany, 10-12 September 2003, 12-19.

[10] Atanassov, K. T. (2012). On Intuitionistic Fuzzy Sets. Springer-Verlag, Heidelberg.

[11] Atanassova, L. C. (1995). Remark on the cardinality of intuitionistic fuzzy sets. Fuzzy Sets and Systems, 75, 399-400.

[12] Bustince, H., Kacprzyk, J., \& Mohedano, V. (2000). Intuitionistic fuzzy generators, applications to intuitionistic fuzzy complementation. Fuzzy Sets and Systems, 144, 485-504. 
[13] De, S. K., Biswas, R., \& Roy, A. R. (2000). Some operations on intuitionistic fuzzy sets. Fuzzy Sets and Systems, 114, 477-484.

[14] De, S. K., Biswas, R., \& Roy, A. R. (2001). An application of intuitionistic fuzzy sets in medical diagnostic. Fuzzy sets and Systems, 117(2), 209-213.

[15] Ejegwa, P. A. (2020). An improved correlation coefficient between intuitionistic fuzzy sets and its applications to real-life decision-making problems. Notes on Intuitionistic Fuzzy Sets, 26(2), 1-14.

[16] Ejegwa, P. A. (2020). Modified and generalized correlation coefficient between intuitionistic fuzzy sets with applications. Notes on Intuitionistic Fuzzy Sets, 26(1), 8-22.

[17] Ejegwa, P. A. (2021). Novel correlation coefficient for intuitionistic fuzzy sets and its application to multi-criteria decision-making problems. International Journal of Fuzzy System Applications, 10(2), 39-58.

[18] Ejegwa, P.A., \& Adamu, I. M. (2019). Distances between intuitionistic fuzzy sets of second type with application to diagnostic medicine. Notes on Intuitionistic Fuzzy Sets, 25(3), 53-70.

[19] Ejegwa, P. A., Akowe, S. O., Otene, P. M., \& Ikyule, J. M. (2014). An overview on intuitionistic fuzzy sets. International Journal of Scientific and Technological Research, $3(3), 142-145$.

[20] Ejegwa, P. A., Akubo, A. J., \& Joshua, O. M. (2014). Intuitionistic fuzzy set and its application in career determination via normalized Euclidean distance method. European Scientific Journal, 10(15), 529-536.

[21] Ejegwa, P. A., \& Onasanya, B. O. (2019). Improved intuitionistic fuzzy composite relation and its application to medical diagnostic process. Notes on Intuitionistic Fuzzy Sets, 25(1), 43-58.

[22] Ejegwa, P. A., \& Onyeke, I. C. (2019). An object oriented approach to the application of intuitionistic fuzzy sets in competency based test evaluation. Annals of Communications in Mathematics, 1(1), 38-47.

[23] Ejegwa, P. A., \& Onyeke, I. C. (2020). Medical diagnostic analysis on some selected patients based on modified Thao et al.'s correlation coefficient of intuitionistic fuzzy sets via an algorithmic approach. Journal of Fuzzy Extension and Applications, 1(2), 130-141.

[24] Ejegwa, P. A., \& Onyeke, I. C. (2021). Intuitionistic fuzzy statistical correlation algorithm with applications to multi-criteria based decision-making processes. International Journal of Intelligent Systems, 36(3), 1386-1407. 
[25] Ejegwa, P. A., Onyeke, I. C., \& Adah, V. (2020). An algorithm for an improved intuitionistic fuzzy correlation measure with medical diagnostic application. Annals of Optimization Theory and Practice, 3(3), 51-66.

[26] Ejegwa, P. A., Uleh, B. S., \& Onwe, E. (2014). Intuitionistic fuzzy sets in electoral system. International Journal of Science and Technology, 3(4), 241-243.

[27] Hatzimichailidis, A. G, Papakostas, G. A., \& Kaburlasos, V. G. (2012). A novel distance measures of intuitionistic fuzzy sets and its application to pattern recognition applications. Technological Educational Inst. of Kavala, Dept. of Industrial Informatics, 65404 Kavala, Greece.

[28] Huawen, L. (2000). Axiomatic construction for intuitionistic fuzzy sets, The Journal of Fuzzy Mathematics, 8(3), 645-650.

[29] Ibrahim, A. M., \& Ejegwa, P. A. (2013). Remark on some operations in intuitionistic fuzzy sets. International Journal of Science and Technology, 2(1), 94-96.

[30] Li, D., \& Cheng, C. (2002). New similarity measures of intuitionistic fuzzy sets and application to pattern recognitions. Pattern Recognition Letters, 23, 221-225.

[31] Szmidt, E. (2014). Distances and Similarities in Intuitionistic Fuzzy Sets, Springer.

[32] Szmidt, E., \& Kacprzyk, J. (1997). On measuring distances between intuitionistic fuzzy sets. Notes on Intuitionistic Fuzzy Sets, 3(4), 1-13.

[33] Szmidt, E., \& Kacprzyk, J. (2000). Distances between intuitionistic fuzzy Sets. Fuzzy Sets and Systems, 114(3), 505-518.

[34] Szmidt, E., \& Kacprzyk, J. (2001). Intuitionistic fuzzy sets in some medical applications. Notes on Intuitionistic Fuzzy Sets, 7(4), 58-64.

[35] Szmidt, E., \& Kacprzyk, J. (2004). Medical diagnostic reasoning using a similarity measure for intuitionistic fuzzy sets. Notes on Intuitionistic Fuzzy Sets, 10(4), 61-69.

[36] Takeuti, G., \& Titani, S. (1984). Intuitionistic fuzzy logic and Intuitionistic fuzzy set theory. Journal of Symbolic Logic, 49, 851-866.

[37] Wang, W., \& Xin, X. (2005). Distance measure between intuitionistic fuzzy sets. Pattern Recognition Letters, 26, 2063-2069.

[38] Ye, J. (2011). Cosine similarity measures for intuitionistic fuzzy sets and their applications. Mathematics and Computer Modelling, 53, 91-97.

[39] Zadeh, L. A. (1965). Fuzzy sets. Information and Control, 8(3), 338-353.

[40] Zeng, W., \& Li, H. (2006). Note on some operations on intuitionistic fuzzy sets. Fuzzy Sets and Systems, 157, 990-991. 